\title{
Influences of Maternal Weight Gain During Pregnancy on Selected Fetal Outcomes
}

\author{
Raguraman Sivalingarajah ${ }^{*}$, Wimalasiri Abeykoon \\ Teaching Hospital Kandy, Kandy, Sri Lanka \\ Email address: \\ sivalingarajahraguraman@gmail.com (R. Sivalingarajah), wimalabeykoon@gmail.com (W. Abeyakoon) \\ ${ }^{*}$ Corresponding author
}

To cite this article:

Raguraman Sivalingarajah, Wimalasiri Abeykoon. Influences of Maternal Weight Gain During Pregnancy on Selected Fetal Outcomes. Journal of Gynecology and Obstetrics. Vol. 7, No. 4, 2019, pp. 120-124. doi: 10.11648/j.jgo.20190704.15

Received: July 11, 2019; Accepted: August 10, 2019; Published: August 23, 2019

\begin{abstract}
Weight gain in pregnant mothers has several influencing factors. Studies have shown that maternal weight gain influenced both maternal and fetal immediate and future outcomes. However, the recommended amount of weight gain for optimum maternal and fetal outcome is still uncertain. A cross sectional descriptive study was carried out at Teaching Hospital Kandy, for a period of one-year to describe the influence of maternal weight gain during pregnancy in selected fetal outcome such birth weight and APGAR score at birth. 425 participants with normal pre gestational BMI $\left(18.5 \mathrm{~kg} / \mathrm{m}^{2}-24.9 \mathrm{~kg} / \mathrm{m}^{2}\right)$ were selected by a systematic random sampling technique. Medical disorders complicating pregnancies, twins, previous miscarriages and fetal abnormalities were excluded. Data was extracted from the antenatal record, bed head ticket and by measuring relevant variables (birth weight and APGAR score). Maternal age distributed from 17 to 43 years (Mean=27.97 years: $\mathrm{SD}=5.72$ years). Maternal height distributed from $125 \mathrm{~cm}$ to $172 \mathrm{~cm}$ (Mean $=154.4 \mathrm{~cm}: \mathrm{SD}=5.83 \mathrm{~cm}$ ). Pre pregnancy BMI distributed from $18.5 \mathrm{~kg} / \mathrm{m}^{2}$ to $24.9 \mathrm{~kg} / \mathrm{m}^{2}$ (Mean=21.67 kg/m $: \mathrm{SD}=2.2 \mathrm{~kg} / \mathrm{m}^{2}$ ). Maternal body weight at delivery distributed from $36 \mathrm{~kg}$ to $116 \mathrm{~kg}$ (Mean=63.8kg: $\mathrm{SD}=11.82$ ). Pregnancy weight gain distributed from $3.5 \mathrm{~kg}$ to $24.5 \mathrm{~kg}$ (Mean=9.03kg: $\mathrm{SD}=3.87$ ). Birth weight distributed from $1.24 \mathrm{~kg}$ to $4.04 \mathrm{~kg}$ (Mean=2.93: $\mathrm{SD}=0.438)$. All exposure parameters had a positive linear correlation with birth weight. Almost all the study participants ( $N=423: 99.5 \%)$ had achieved an APGAR score of $>7$ within 10 minutes of birth. In conclusion, maternal weight gain does not affect the birth weight of the newborn and no conclusion is derived on maternal weight gain causing any hypoxic situation at the time of birth. These findings are only applicable to normal pregnancies due to the exclusion criteria. Further studies are recommended with a larger sample size and a prospective cohort design with continuous follow up during the antenatal period.
\end{abstract}

Keywords: Body Mass Index, Maternal Weight Gain, Birth Weight, APGAR Score

\section{Introduction}

Weight gain during pregnancy occurs due to several factors such as products of conception, maternal tissue such as uterus and breast and to a greater extent due to maternal fat stores. There are individual variations in maternal weight gain during pregnancy among mothers. Maternal weight gain influences both maternal and fetal immediate and future outcome.

Throughout the last fifty years, recommended amount of weight gain during pregnancy was uncertain. About five decades ago obstetric text books mentioned that excessive weight gain during pregnancy is highly undesirable. It said that weight gain should be curtailed to $12 \mathrm{~kg}$ (25lb) and this recommendation lead to lot of criticism [1].

Earlier, Institute of Medicine (IOM) of National Academy of Sciences formulated recommendations for maternal weight gain during pregnancy. But these were revised in 2009 and published gestational weight gain guidelines were based on pre-pregnancy BMI. [2-3]. BMI categories were considered as recommended by the WHO underweight, normal weight, overweight and obese [4]. The WHO also has prioritized achievement of ideal BMI before conception and prevent excess weight gain [5].

Mothers should take a balanced nutritious diet to achieve the optimal weight gain during pregnancy. Some believe that they should double their food intake in order to supply additional energy to the fetus. With this misbelief they end up with excessive weight gain, leading to many complications [6]. 
In Sri Lanka, maternal weight gain during pregnancy ranged from $7 \mathrm{~kg}-8 \mathrm{~kg}$ and around $30 \%$ of the mothers have less than 18.5 BMI during their first trimester [7]. A Recent systematic review has shown Indian and south Asian women's weight gain during pregnancy lower than IOM recommendation [8]. In Sri Lanka, antenatal pregnancy record monitors monthly weight gain and a graph is available to plot the weight gain $(\mathrm{kg})$ against gestational age (weeks). This monitoring system functions at a field level and optimal weight gain can be identified during pregnancy by observing the graph. According to a circular issued by the Director General of Health Services Sri Lanka, height and weight should be measured in all mothers presenting to the antenatal clinic for the first visit and these should be marked in the pregnancy record. (H 512 A and B). BMI should be calculated in all mothers at the booking visit before 12 weeks of gestational age. Standard and Validated BMI charts are issued by the Family Health Bureau to calculate the Body Mass Index. BMI and weight gain are marked in the weight gain chart with the pregnancy record.

Kandy is the main district of the Central Province. It has an area of $1906 \mathrm{~km} 2$ with an estimated population of 1.3 million. There are nearly 12,000 deliveries per year in this district. The second-largest hospital in Sri Lanka is situated in Kandy and it receives about 15,000 clinic patients per year [7]. Approximately $85 \%$ of deliveries in Sri Lanka occur under tertiary care facilities [9].

The Objectives of this study were to find the association between the birth weight of the baby and maternal weight gain during pregnancy and the association between the APGAR score of the baby at birth and maternal weight gain during pregnancy.

\section{Materials and Methods}

This was a descriptive cross-sectional study and it was carried out at the Teaching Hospital Kandy, a tertiary care hospital in Sri Lanka. It was done for a period of one year starting from July 2014.

Pregnant mothers presented to the antenatal clinic at the hospital were selected as the target population. Clinic attendance register was used as the sampling frame and systematic random sampling technique was applied. After applying eligibility criteria and with informed written consent, every third mother was selected for the study. Selection was done at the $1^{\text {st }}$ clinic visit of the participant during the $1^{\text {st }}$ trimester of their pregnancy. 425 study units were followed up completely who ended their pregnancy with a live birth. A specific code number was given to all the selected mothers and a sticker with a specific colour containing the code number was included in their notes. During the follow up clinic visits, participants were identified by using this sticker and the code number.

Mothers with normal pre gestational BMI (18.5-24.9) were included and mothers with below mentioned conditions were excluded from the study; mothers with already diagnosed medical diseases such as GDM, PIH, Heart disease, Epilepsy, Anemia and Connective tissue disorders, multiple pregnancies, mothers with more than two miscarriages during first or second trimester, mothers with past history of recurrent preterm delivery and mothers with antenatal diagnosis of fetal anomaly.

Data were extracted from mother's antenatal record (H512), bed head ticket and by measuring relevant variables. Weight of the pregnant mothers was measured at the antenatal clinic on her first visit no later than the first 10 weeks of gestation. Subsequently, mother's weight was measured and documented monthly. Weighing scale was calibrated daily due to frequent use. This led to a higher accuracy of the measurements. Gestational age was calculated either by early ultrasonography (dating scan). Baby's birth weight was measured immediately after delivery.

Selected fetal outcomes, such as the APGAR score $(>7,7$ to $4,<4$ ) which was measured within five minutes and ten minutes of delivery were recorded.

Data was analyzed by using Statistical Package for Social Sciences (SPSS); version 25.0. Ethical clearance for the study was obtained from the Ethical Review Committee, Teaching Hospital Kandy.

\section{Results}

Table 1 describes the distribution of maternal age, maternal height and pre pregnancy body mass index of the participants. Maternal age ranged from 17 to 43 years (Mean=27.97 years: $\mathrm{SD}=5.72$ ). Maternal height ranged from $125 \mathrm{~cm}$ to $172 \mathrm{~cm}$ (Mean=154.4cm: $\mathrm{SD}=5.83 \mathrm{~cm}$ ). Pre pregnancy $\mathrm{BMI}$ ranged from 18.5 to 24.9 in the study sample (Mean $=21.67 \mathrm{~kg} / \mathrm{m}^{2}$ : $\left.\mathrm{SD}=2.2 \mathrm{~kg} / \mathrm{m}^{2}\right)$.

Table 2 demonstrates the distribution of maternal weight at delivery, pregnancy weight gain and, birth weight of the new born. Maternal body weight at delivery distributed from $36 \mathrm{~kg}$ to $116 \mathrm{~kg}$ (Mean=63.8kg: SD=11.82kg). Pregnancy weight gain ranged from $3.5 \mathrm{~kg}$ to $24.5 \mathrm{~kg}$ during the antenatal period (Mean=9.03kg: $\mathrm{SD}=3.87 \mathrm{~kg}$ ). Birth weight of the new born distributed from $1.24 \mathrm{~kg}$ to $4.04 \mathrm{~kg}$ (Mean=2.95kg: $\mathrm{SD}=0.438 \mathrm{~kg}$ ).

Figures $1 \& 2$ displayed the distribution of maternal weight gain and birth weight of newborn. Majority of the participants achieved $5 \mathrm{~kg}-10 \mathrm{~kg}$ of weight gain during their pregnancy period $(\mathrm{N}=211: 49.6 \%)$. Majority of children's birth weight was within the range from 2.5 to $3.5 \mathrm{~kg}(\mathrm{~N}=332: 78.1 \%)$.

Table 1. Distribution of maternal height, maternal age and pre pregnancy Body Mass Index of the participants

\begin{tabular}{llll}
\hline & Pre pregnancy BMI & Maternal Age (years) & Maternal Height (cm) \\
\hline Mean & 21.67 & 27.97 & 154.4 \\
Median & 21.5 & 28.0 & 154.0 \\
Standard Deviation & 2.2 & 5.72 & 5.83 \\
Minimum value & 18.5 & 17 & 125 \\
Maximum Value & 24.9 & 43 & 172 \\
\hline
\end{tabular}


Table 2. Distribution of maternal weight at delivery, pregnancy weight gain and birth weight of the participants.

\begin{tabular}{llll}
\hline & Maternal weight $(\mathbf{k g})$ & Pregnancy weight gain (kg) & Birth weight (kg) \\
\hline Mean & 63.8 & 9.03 & 2.93 \\
Median & 63.0 & 8.7 & 2.95 \\
Standard Deviation & 11.82 & 3.87 & 0.438 \\
Minimum value & 36 & 3.5 & 1.24 \\
Maximum Value & 116 & 24.5 & 4.04 \\
\hline
\end{tabular}

Table 3. Correlation of maternal pregnancy weight gain with Anthropometric parameters.

\begin{tabular}{llll}
\hline & Maternal pregnancy weight gain & & \\
\cline { 2 - 4 } & Pearson's (r) & P value & N \\
\hline Pre - pregnancy BMI & -.218 & $<0.001$ & 425 \\
BMI at 10 weeks & -.223 & $<0.001$ & 425 \\
Weight at 10 weeks & -.245 & $<0.001$ & 425 \\
Maternal age & -.145 & 0.003 & 425 \\
Maternal Height & 106 & 0.029 & 425 \\
\hline
\end{tabular}

Table 3 shows the association of maternal pregnancy weight gain with several anthropometric parameters. All selected parameters except maternal height, negatively correlated with birth weight. Only maternal height has shown a positive correlation $(\mathrm{r}=0.106)$. All the selected parameters had a significant association with birth weight $(\mathrm{p}<0.05)$

Table 4. Association of Maternal parameters with Birth weight of new born.

\begin{tabular}{llll}
\hline & Birth Weight & & Significant level \\
\cline { 2 - 4 } & r & P value & $\mathrm{P}<0.01$ \\
Maternal height & 0.194 & 0.000 & $\mathrm{P}<0.05$ \\
Maternal Age & 0.119 & 0.014 & $\mathrm{P}<0.01$ \\
Pre-Pregnancy BMI & 0.164 & 0.001 & $\mathrm{P}<0.01$ \\
Maternal weight at delivery & 0.317 & 0.000 & $\mathrm{P}>0.05$ \\
Pregnancy weight gain & 0.081 & 0.065 & \\
\hline
\end{tabular}

\section{Maternal weight gain}

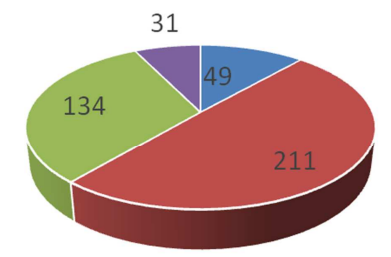

$=<\mathrm{kg}=5-10 \mathrm{~kg}=10-15 \mathrm{~kg} \quad=>15 \mathrm{~kg}$

Figure 1. Distribution of maternal weight gain.

\section{Birth weight}

37

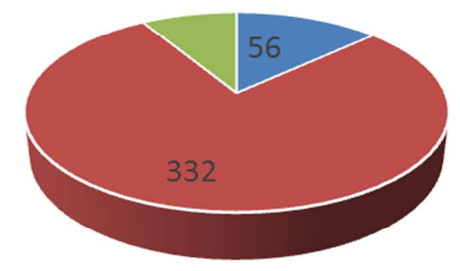

- $<2.5 \mathrm{~kg} \quad=2.5-3.5 \mathrm{~kg}=>3.5-4 \mathrm{~kg}$

Figure 2. Distribution of birth weight of newborn.
Table 4 shows the correlation figures between exposure variables and birth weight. All parameters have a positive linear correlation with birth weight. All the correlation coefficient values except pregnancy weight gain were significant with the birth weight. Almost all the study participants $(\mathrm{N}=423: 99.5 \%)$ had achieved an APGAR score of $>7$ within 10 minutes of birth.

\section{Discussion}

The goal of this study was to find out the effect of maternal weight gain during pregnancy towards the outcome of the newborn baby. According to the findings of the study there is a significant association between the birth weight of the baby and the parameters such as maternal age, height pre pregnancy BMI, and maternal weight at delivery. But the association between the birth weight and the weight gain during pregnancy is not significant. But the $p$ value is 0.06 and it is closer to the probability cut off value which is 0.05 .

In this study sample, possibility of this relationship between pregnancy weight gain and the birth weight is $6 \%$. Therefore according to the available statistics we cannot come to a conclusion that there is an association between the birth weight of the baby and the weight gain during pregnancy.

According to the study done by SchollTO et al. 1991, they concluded that birth weight may be indirectly moderated by weight gain during pregnancy [10].

A negative association was identified between the gestational weight gain of mothers and the sub-optimal birth 
weight of the new born in a study conducted by using 1120 mothers from America and some parts of Africa from year 1990 to 2000. These findings are compatible with the current study findings [11].

A significant association was noted between the low pregnancy weight gain and low birth weight in a study done in America before the above-mentioned study [12]. Recent studies has explained positive association with suboptimal maternal weight during pregnancy and low birth weight [1314]. During the current study, weight gain during each trimester was not considered separately. Also, there were no opportunities to predict other factors associated with low birth weight during the study. It may be possible to study the risk factors associated with low birth weight by using a case control study design in future studies. According to the systematic review done by Meera Vishwathma et al in 2008, gestational weight gain affects major factors of the new born. Namely preterm birth, low birth weight, macrosomia and appearing too large or small for gestational age. These results should be studied independently and recommendations should be suggested accordingly [15]. That was not possible in the present study due to time constraints. $38 \%$ of participants in the present study had pregnancy weight gain more than $10 \mathrm{~kg}$. Out of that $18.7(\mathrm{~N}=31)$ had a weight gain more than $15 \mathrm{~kg}$.

A hypothesis of strong relationship between excessive weight gain and long-term obesity was developed by Rooney et al according in 2002 [16]. South Asian studies have clearly explained the positive correlation between excessive maternal weight gain and short term and long term complications in new born such as large for dates, childhood obesity and Diabetic mellitus in adulthood [17-18].

In this study, there was a strong negative association between pregnancy weight gain and birth weight. But nearly one third of the study sample had an excessive weight gain. It is possible to identify the relationship and associated factors between pregnancy weight gain and long-term obesity by conducting a follow-up study on these participants who had excessive weight gain. But it will be a highly expensive and less feasible with significant attrition bias.

There were $20.2 \%$ of Sri Lankan pregnant mothers with low Body Mass Index in 2015. 11.36\% of live births had low birth weight but, it was very hard to find a study or data describing the outcome of mothers who had low Body Mass Index or nutritional status of mothers who had babies born with low birth weight in Sri Lanka [19].

In the present study, the percentage of mothers who had Body Mass Index less than 18.6 was $19.7 \%(\mathrm{~N}=84)$. That value was compatible with the national figures. However, in this study sample, mothers with BMI less than 18.6 were not considered. Therefore, it is possible to develop a hypothesis that there is no pathological deviation of birth weight of babies with pregnancy weight gain or pre pregnancy nutritional status. It would be a newly generated study area introduced by this study, which will be studied in detail with well-developed scientific methodology.

In this study, weight gain was measured prospectively.
Factors such as measuring techniques, skills of health care workers, and the validity of the measuring scales were carefully considered during this study. It positively affected the internal validity of the study. Data of the participants who were not followed up were not considered. Therefore, a certain degree of attrition bias was incorporated with the study findings. And the potential confounding factors for maternal weight gain such as nutrition, occupation, and stress levels were not considered. Randomization was the only practical method that could be used to minimize the effects of the confounding factors. It was fulfilled by using the simple random sampling technique. In future studies, potential confounding factors should be addressed properly.

The APGAR score is a simple assessment of a baby's condition at birth which helps to determine whether the new born is ready to meet the world without additional medical assistance. There are numerous factors that can influence the APGAR score such as maternal sedation or anesthesia, labour duration, gestational age, trauma and inter observer variability [12]. Incidents closer to the delivery affect the APGAR score significantly. However, in this study APGAR score did not change according to the variables used in this study.

\section{Conclusion}

This study concluded that maternal weight gain does not affect the birth weight of the new born in normal pregnancies. No conclusion is derived on maternal weight gain causing any hypoxic situation at the time of birth. Again this is only applicable to normal pregnancies due to exclusion criteria. Further studies could be recommended with a larger sample size and a prospective cohort design with continuous follow up during the antenatal period.

\section{References}

[1] Eastman N, Hellman L. William's obstetrics. $13^{\text {th }}$ Ed. New York: Appleton-Century-Crofts, 1966: 326.

[2] Z. M. Ferraro, F. Contador, A. Tawfiq, K. D. Adamo, L. Gaudet. Gestational weight gain and medical outcome of pregnancy: Obstetric medicine. 2015; 8 (3): 133-137.

[3] U. Radhakrishnan, G. Kolar, P. K. Nirmalan. Cross sectional study of gestational weight gain and perinatal outcomes in pregnant women at a tertiary center in Sothern India. Journal of Obstetrics and Gynaecology Research. 2014; 40 (1): 25-31.

[4] Institute of medicine. Weight gain during pregnancy: reexamining guidelines. Washington, DC: National academic press; 2009.

[5] World health organization, WHO recommendation on antenatal care for a positive pregnancy experience, Geneva, Switzerland, 2016.

[6] Lumbiganon P, Lapaiboon M, Glumezoglu M, Zousa JP, taneepa Nichkul S, Ruyan $\mathrm{p}$ et al. Method of delivery and pregnancy out comes in Asia: The global survey on maternal and perinatal health 2007-2008. Lancet 2010; 375: 490-499. 
[7] Ministry of health. Health service delivery: strengthening of maternal health services. Sri Lanka, ministry of health; 2008.

[8] A. Priyanka, A. Bani Tamber. Gestational Weight Gain amoung Healthy Pregnant Women from Asia in Comparison with Institute of Medicine (IOM) Guidelines 2009: A systemic review. Journal of Pregnancy. 2019; Vol 2019, article ID 3849596, 10 pages.

[9] Annual health bulletin, teaching hospital kandy. 2011.

[10] Scholl TO, Hediger ML, Khoo CS, Healey MF, Rawson NL. 1991: Maternal weight gain, diet and infant birth weight: correlations during adolescent pregnancy. Journal of Clinical Epidemiology. 1991; 44 (4-5): 423-8.

[11] Abram B, Altman SL, Pickett KE. Pregnancy weight gain: still controversial. The American journal of clinical nutrition. 2000; 71 (5): 1233-1241.

[12] Abram B, Newman V, Key T, Parker J. Maternal weight gain and premature delivery. Obstetrics and gynecology. 1998; 74 (4): $577-583$.

[13] M. F. Young, P. Hong Nguyen, o. y. Addo et.al. Timing of gestatinal weight gain on fetal growth and infant size at birth in Vietnam. PLoS ONE. 2017; 12 (1): p0170192.

[14] R. F. Goldstain, S. K. Abell, S. Ranasinha et.al. Association of gestational weight gain with maternal and infant outcomes. Journal of the American Medical Association. 2017; 317 (2); 2207.
[15] Meera Viswanathan, Anna Maria Siega, Merry-K Moos, eierlein, MS, MPH, Sunni Mumford Julie Knaack, Patricia Thieda LJL and KNL. Outcomes of maternal weight gain. Technology Assesments. New York. New York; 2008.

[16] Rooney BL, Schauberger CW. Excess Pregnancy Weight Gain and Long-Term Obesity: One Decade Later. 2002; 100 (2): $245-52$.

[17] R. Pal, M. Maiti, B. Roychoudhury, P. Sanyal, B. chowdhury. Association of pregestational BMI and antenatal weight gain with pregnancy outcome: A Prospective Observational Cohort Study. International Journal of Women's Health and Reproductive Sciences. 2017; 5 (1): 37-40.

[18] L. Xiao, G. Ding, A. Vinturache et.al. Association of maternal pre-pregnancy body mass index and gestational weight gain with birth outcomes in Shanghai, China. Scientific Reports. 2017; 7 (1): 41073.

[19] Beaureu FH. Statistics [Internet]. [cited 2017 Jan 10]. Available from: http://fhb.health.gov.lk/web/index.php?option=com_statistics \&view=islandwideallresult\&Itemid=134\&lang=en. 